\title{
Computação com Mídias na Aprendizagem de Programação Orientada a Objetos em um Curso Técnico de Informática
}

\author{
Ayala Lemos Ribeiro ${ }^{1}$, Ivanaldo Cerqueira Carvalho ${ }^{1}$, \\ Luis Gustavo de Jesus Araújo ${ }^{1}$, Roberto Almeida Bittencourt ${ }^{1}$ \\ ${ }^{1}$ UEFS - Universidade Estadual de Feira de Santana \\ Av. Transnordestina, s/n, Novo Horizonte \\ Feira de Santana - BA, Brasil - 44036-900 \\ \{ayalaedavi, ivanfsa, luisaraujo.ifba\}@gmail.com, roberto@uefs.br
}

\begin{abstract}
Problems related to learning programming in computing programs contribute to demotivation, failure and early dropout of these programs. This paper aims to investigate how the media computation approach, using the JES environment and the Python language, may affect the students' learning in a vocational IT program in a public high school in Brazil. Results suggest that the approach using image manipulation allowed greater behavioral, emotional and cognitive engagement of the students, and facilitated learning of programming concepts.
\end{abstract}

Resumo. Problemas relacionados à aprendizagem de programação em cursos de computação contribuem para desmotivação, reprovação e abandono precoce destes cursos. Este artigo investigou como o uso de mídias, apoiado pelo ambientes JES e pela linguagem Python, pode afetar a aprendizagem de estudantes de uma escola pública de ensino médio integrado com a educação profissional em informática. Os resultados sugerem que a abordagem utilizando manipulação de imagens permitiu um maior engajamento comportamental, emocional e cognitivo dos estudantes em relação à disciplina, facilitando a aprendizagem dos conceitos de programação.

\section{Introdução}

Desmotivação, reprovação e evasão são problemas constantes enfrentados por cursos da área de Computação, especialmente nas disciplinas de algoritmos e programação. Estas disciplinas costumam ter altos índices de evasão e reprovação, dificultando ou impedindo a continuidade dos alunos no curso. Dentre possíveis fatores que contribuem para esta situação, pode-se elencar: i) falta de capacidade de abstração e raciocínio lógico para desenvolver soluções algorítmicas; ii) falta de motivação do estudante, que, muitas vezes, encara a disciplina como um grande obstáculo a ser superado; e iii) abordagem de ensino instrucionista, que pode não despertar o interesse do estudante [Hinterholz 2009].

É relativamente comum introduzir programação através do paradigma imperativo e, posteriormente, apresentar o paradigma orientado a objetos nas disciplinas de programação dos cursos de computação. Esta transição cria um problema adicional, pois provoca um conflito cognitivo nos aprendizes, geralmente demorado de resolver [Bittencourt et al. 2013]. Associada aos fatores anteriores, esta organização curricular aumenta a complexidade na aquisição de habilidades de programação, gerando 
VI Congresso Brasileiro de Informática na Educação (CBIE 2017)

Anais dos Workshops do VI Congresso Brasileiro de Informática na Educação (WCBIE 2017)

desmotivação e, consequentemente, dificultando a aprendizagem e a retenção do conhecimento.

A adoção de abordagens lúdicas para facilitar a aprendizagem de programação é uma das iniciativas tomadas para solucionar esse problema. O desenvolvimento de jogos auxilia nas disciplinas de programação, servindo como estímulo, realçando o papel educacional e pedagógico dos jogos como ferramenta de aprendizagem [Quadros et al. 2012]. Ferramentas como Greenfoot, Alice e Scratch são utilizadas para auxiliar o desenvolvimento do raciocínio lógico com a criação de jogos [Oliveira et al. 2016]. Em outra linha, a manipulação de mídias digitais também possibilita uma aprendizagem mais lúdica. Em 2003, o Georgia Tech ofereceu um novo curso introdutório com foco em computação e utilizando o contexto de mídias, cujo objetivo era motivar os estudantes de cursos de áreas diferentes da área de TI [Guzdial et al. 2005]. Foram introduzidos conceitos de programação e computação através da criação de filtros em imagens semelhantes ao utilizados em programas como Photoshop, da manipulação de amostras para reverter e dividir sons, além da criação de páginas Web e da geração de animações. Este novo formato de disciplina apresentou melhores taxas de sucesso e aumentou a sensação de relevância para a carreira profissional e para as aspirações pessoais dos estudantes.

Adotamos a manipulação de mídias em nosso contexto de ensino técnico de nível médio pois entendemos que seu uso permite aprender conceitos e desenvolver habilidades básicas de programação orientada a objetos (POO), oferecendo aos estudantes uma experiência interessante e divertida, adequada à idade dos estudantes. A abordagem construcionista para aprendizagem afirma que as pessoas aprendem particularmente bem quando estão envolvidas na construção de um artefato público que é pessoalmente significativo [Papert 1980] Por outro lado, encontramos poucos trabalhos utilizando a abordagem com mídias no Brasil, o que motivou a realização desta investigação.

O objetivo deste estudo foi explorar como a abordagem de programação pela manipulação de imagens, i.e., computação com mídias, utilizando o ambiente JES e a linguagem Python, pode afetar a aprendizagem de estudantes de uma escola pública de ensino médio integrado com a educação profissional em Informática de nível técnico através de uma abordagem de estudo de caso qualitativo.

Este artigo está organizado como a seguir. A Seção 2 apresenta os trabalhos relacionados, seguida pela Seção 3, que apresenta a metodologia de pesquisa. A Seção 4 descreve os resultados. A discussão está descrita na Seção 5. Por fim, expomos nossas conclusões na Seção 6.

\section{Trabalhos Relacionados}

A adoção de um contexto baseado na manipulação de mídias para disciplinas introdutórias em programação (CS1) em uma das maiores universidades dos EUA já foi descrita previamente [Simon et al. 2010]. Os autores realizam um comparativo de CS1 com estudantes de computação utilizando o método tradicional com a mesma disciplina utilizando computação com mídias. São analisadas questões relacionadas às competências requeridas dos estudantes, com foco mais na resolução de problemas e menos na sintaxe da linguagem através de tarefas de programação e avaliações. Os resultados demonstram, ao comparar o sucesso do estudante analisado através de taxas de aprovação e de retenção um ano depois, percentuais significativamente maiores com a utilização da abordagem de 
VI Congresso Brasileiro de Informática na Educação (CBIE 2017)

Anais dos Workshops do VI Congresso Brasileiro de Informática na Educação (WCBIE 2017)

manipulação de mídias.

Santana et al. (2017) apresentam um estudo utilizando contextos de mídias no ensino de programação, investigando como uma abordagem de ensino de programação para non-majors, que faz uso do ambiente Scratch e da linguagem de programação Python com a biblioteca Turtle e com o ambiente de desenvolvimento JES, influencia na motivação dos estudantes. Para avaliar as diversas dimensões de motivação, utilizou-se o modelo de motivação ARCS (Atenção, Relevância, Confiança e Satisfação). Os resultados mostram que o ambiente Scratch proporciona o aumento da motivação dos estudantes. Em contrapartida, com a ferramenta JES no contexto de imagens, não se conseguiu motivar os estudantes satisfatoriamente. Todavia, algumas complicações podem ter impactado no resultado final, demandando investigação posterior.

Um outro trabalho avalia a aprendizagem de programação com estudantes do nono ano da educação fundamental, utilizando uma abordagem em espiral com manipulação de imagens associada ao ambiente JES e à linguagem Python [Araujo et al. 2017]. Foram avaliados os aspectos motivacionais do uso de manipulação de imagens e as impressões dos estudantes sobre programação e computação. Os resultados demonstram que a programação em pares, o ambiente JES e a manipulação de imagens são elementos que estimulam a criatividade dos estudantes, além da mudança na percepção negativa que os estudantes têm em relação à computação.

\section{Metodologia}

O presente trabalho adotou o estudo de caso de natureza essencialmente qualitativa como estratégia de investigação. O objetivo, nesta metodologia, é analisar e compreender o fenômeno vivido através das experiências humanas em um contexto real.

As questões de pesquisa que orientaram este estudo foram:

1. Como a abordagem de uso de mídias afeta o aprendizado de conceitos de POO?

2. De que maneira a abordagem utilizada impacta no engajamento dos estudantes em relação as atividades propostas?

\subsection{Participantes}

O trabalho contou com a participação de 11 alunos do terceiro ano do ensino médio integrado com a educação profissional em Informática, sendo seis meninos e cinco meninas, com idade média de 17,2 anos (desvio padrão 1,27). Em 2017, todos estavam matriculados em uma escola estadual em Feira de Santana, Bahia. Antes de iniciarmos a pesquisa, um termo de consentimento livre e esclarecido (TCLE) foi entregue aos participantes, permitindo a participação no estudo e garantindo anonimato dos participantes e confidencialidade dos dados. Os responsáveis pelos estudantes concordaram com a participação deles na pesquisa.

\subsection{Ferramenta}

Na busca de alternativas que pudessem minimizar as dificuldades do ambiente de desenvolvimento e facilitar a interação dos estudantes com a ferramenta, escolhemos a linguagem Python no contexto de mídias com o ambiente JES - Jython Environment for Students. A linguagem Python, criada por Guido Van Rossum, gratuita e de código aberto, 


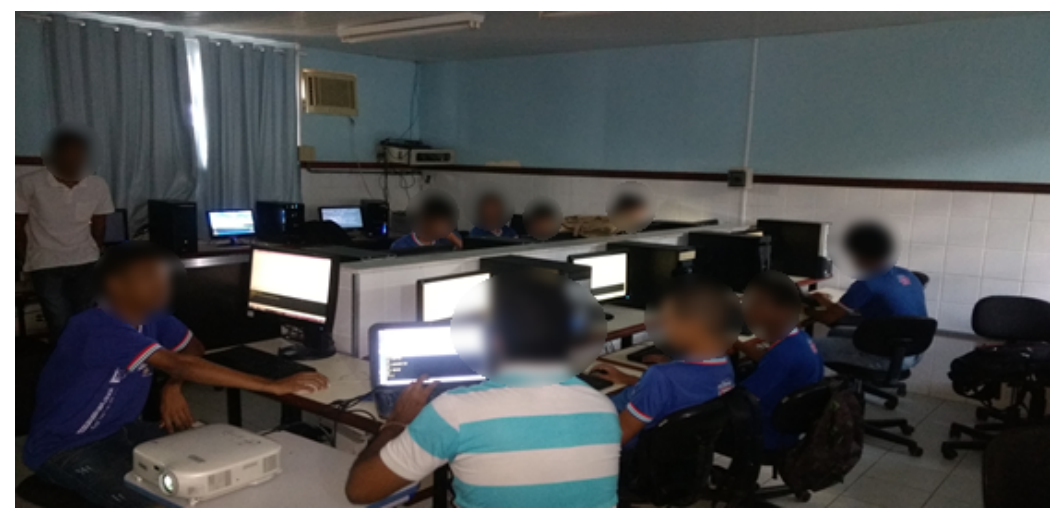

Figura 1. Laboratório de Informática

Tabela 1. Visão geral do módulo Computação com Mídias II

\begin{tabular}{|r|l|}
\hline Local & Colégio Estadual Dr. Jair Santos Silva \\
\hline Curso & $\begin{array}{l}\text { Ensino Médio integrado com Educação } \\
\text { Profissional em Informática }\end{array}$ \\
\hline Ferramenta & Ambiente JES e linguagem Python \\
\hline Conteúdo & $\begin{array}{l}\text { classes, atributos, métodos, } \\
\text { loops, condicionais, } \\
\text { composição, herança }\end{array}$ \\
\hline Desafios & $\begin{array}{l}\text { Efeitos em imagens: livre, degradê, } \\
\text { escala de cinza, matiz, preto e branco }\end{array}$ \\
\hline Duração & \begin{tabular}{l}
12 horas \\
\hline
\end{tabular} \\
\hline
\end{tabular}

propõe abstrair as possíveis dificuldades enfrentadas com a linguagem e direciona o estudante para o aprendizado da programação. Apresenta uma sintaxe concisa e clara: seus blocos de códigos são definidos através de indentação, possui tipagem dinâmica, permite a fácil leitura do código e exige poucas linhas de código quando comparada com outras linguagens. $O$ interpretador permite demonstração rápida e interativa de conceitos de programação fornecendo feedback imediato e de fácil compreensão sobre possíveis erros. Python é uma linguagem que ajuda iniciantes a construírem algoritmos de forma mais simples, com acesso a materiais de apoio de forma gratuita [Grandell et al. 2006].

\subsection{Planejamento}

O curso foi planejado em dois módulos: Computação com Mídias I e Computação com Mídias II. No primeiro módulo, os estudantes tiveram um contato com conceitos básicos de programação orientada a objetos. O objetivo foi apresentar, através da manipulação de imagens, conceitos relacionados à programação e ao paradigma orientado a objetos utilizando o ambiente JES e a linguagem Python. Neste módulo, foram apresentados como os computadores entendem a codificação (linguagem), o ambiente JES, tipos de dados (string, integer, float, Picture, Sound), midias digitais (imagem, som, vídeo), pixels, padrão RGB, classes, objetos e alguns métodos da classe Picture.

O modulo Computação com Mídias II, objeto do presente estudo, foi planejado com uma carga horária de 12 horas e realizado no período compreendido entre maio e 
VI Congresso Brasileiro de Informática na Educação (CBIE 2017)

Anais dos Workshops do VI Congresso Brasileiro de Informática na Educação (WCBIE 2017)

julho de 2017, conforme descrição na Tabela 1. Sua extensão se deu devido ao recesso junino das aulas no período de 15 dias. As aulas ocorreram em um laboratório de informática com sete computadores disponíveis (ver Figura 1). Por isso, algumas máquinas eram compartilhadas por duas pessoas. Contou com a colaboração de dois tutores, um monitor, e dois observadores.

O módulo foi dividido em quatro dias, com três horas-aula em cada dia, e obedeceu ao seguinte planejamento de aulas: i) Aprofundando conceitos sobre Classes e Objetos e criando suas Classes; ii) Usando Condicionais e passagem de parâmetros no Construtor e criando a classe Efeito; iii) Usando Composição; iv) Usando Herança. Os objetivos, conteúdos e as atividades propostas estão descritos na Tabela 2.

Tabela 2. Planejamento do módulo Computação com Mídias II

\begin{tabular}{|c|l|l|l|}
\hline$\#$ & Objetivo & Conteúdo & Atividade \\
\hline $\mathbf{0 1}$ & $\begin{array}{l}\text { Aprofundar conhecimentos } \\
\text { sobre objetos e classes }\end{array}$ & $\begin{array}{l}\text { Conceito de POO, classes } \\
\text { e objetos }\end{array}$ & $\begin{array}{l}\text { Criar aplicações } \\
\text { matemáticas } \\
\text { utilizando classes } \\
\text { e objetos }\end{array}$ \\
\hline $\mathbf{0 2}$ & $\begin{array}{l}\text { Construir conhecimentos } \\
\text { sobre construtores, } \\
\text { parâmetros e aprofundar } \\
\text { conhecimentos sobre } \\
\text { estruturas condicionais }\end{array}$ & $\begin{array}{l}\text { Construtores, parâmetros, } \\
\text { métodos e condicionais }\end{array}$ & $\begin{array}{l}\text { Remodelar as classes } \\
\text { anteriores utilizando } \\
\text { parâmetros e criar } \\
\text { métodos de efeitos com } \\
\text { condicionais }\end{array}$ \\
\hline $\mathbf{0 3}$ & $\begin{array}{l}\text { Construir conhecimentos } \\
\text { sobre composição e } \\
\text { aplicá-los à manipulação } \\
\text { de imagens }\end{array}$ & $\begin{array}{l}\text { Conceitos de POO } \\
\text { (composição), repetição, } \\
\text { seleção e funções do JES }\end{array}$ & $\begin{array}{l}\text { Utilizar composição em } \\
\text { classes para criar } \\
\text { efeitos em imagens }\end{array}$ \\
\hline $\mathbf{0 4}$ & $\begin{array}{l}\text { Construir conhecimentos } \\
\text { sobre herança e aplicá-los à } \\
\text { manipulação de imagens }\end{array}$ & $\begin{array}{l}\text { Conceitos de POO (herança) } \\
\text { repetição, seleção, } \\
\text { funções do JES }\end{array}$ & $\begin{array}{l}\text { Criar uma classe que } \\
\text { herda métodos e } \\
\text { atributos da classe } \\
\text { Picture }\end{array}$ \\
\hline $\mathbf{0 5}$ & $\begin{array}{l}\text { Construir conhecimentos } \\
\text { sobre entrada e saída e } \\
\text { aplicá-los à manipulação } \\
\text { de imagens }\end{array}$ & $\begin{array}{l}\text { Herança, funções do } \\
\text { JES (addText), repetição, } \\
\text { instruções de entrada } \\
\text { e saída }\end{array}$ & $\begin{array}{l}\text { Criar uma classe } \\
\text { para geraçãa de } \\
\text { Memes }\end{array}$ \\
\hline $\mathbf{0 6}$ & $\begin{array}{l}\text { Aplicar conhecimentos } \\
\text { construídos no bloco }\end{array}$ & $\begin{array}{l}\text { Composça, sobrecarga, } \\
\text { funções do JES (repaint), } \\
\text { repetição, seleção e } \text { arrays }\end{array}$ & $\begin{array}{l}\text { Criar uma classe } \\
\text { para criação de } \\
\text { GIF animado }\end{array}$ \\
\hline $\mathbf{0 7}$ & $\begin{array}{l}\text { Aplicar conhecimentos } \\
\text { construídos no bloco }\end{array}$ & $\begin{array}{l}\text { Todos os conteúdos } \\
\text { e conceitos apresentados }\end{array}$ & Avaliação escrita \\
\hline
\end{tabular}

\subsection{Coleta e Análise dos Dados}

Utilizamos observações das aulas, entrevistas e diários de bordo como instrumentos de pesquisa qualitativa. As observações foram realizadas nas aulas, seguindo protocolo de observação, realizando anotações baseadas no objetivo e questões da pesquisa, identificando o comportamento dos estudantes quando da apresentação dos conteúdos, na interação entre eles e deles com a ferramenta. Além das observações, ao final do curso, fizemos entrevistas semiestruturadas através de um roteiro de perguntas previamente elaboradas. No decorrer das entrevistas, eram inseridas novas interrogativas quando necessário. Os diários de bordo registraram as percepções dos tutores imediatamente após a aula.

Os dados coletados das observações, diários de bordo e entrevistas foram analisados pelo método de análise de conteúdo, seguindo um procedimento de codificação, inicialmente aberta e em seguida axial, gerando categorias e escrevendo memorandos das 
VI Congresso Brasileiro de Informática na Educação (CBIE 2017)

Anais dos Workshops do VI Congresso Brasileiro de Informática na Educação (WCBIE 2017)

categorias como resultados da análise. A seguir, utilizamos os códigos de E1 a E11 para identificar os estudantes, e $\mathrm{O} 1$ e $\mathrm{O} 2$ para identificar os observadores.

\section{Resultados}

Agrupamos os resultados baseados nas observações, entrevistas e diários de bordo em três temas apresentados a seguir: Abordagem, Aprendizagem de Conceitos de POO e Engajamento.

\subsection{Abordagem}

A turma já havia tido contato prévio com programação na disciplina de Linguagem de Programação I no ano anterior, quando a abordagem utilizada foi o paradigma imperativo e a ferramenta, o VisuAlg. No currículo da disciplina de Linguagem de Programação II, a abordagem previa inicialmente o ensino do paradigma orientado a objetos utilizando o método tradicional, contemplando o uso da linguagem de programação Java e o ambiente de desenvolvimento NetBeans. Mudamos os planos e adotamos o contexto de manipulação de mídias com a linguagem Python apoiada pela ferramenta JES.

Ao compararmos o uso de mídias para programar em um paradigma orientado a objetos com a abordagem tradicional utilizando o paradigma imperativo, observamos que os relatos demonstraram mais envolvimento dos alunos, apesar da complexidade de abstração e da dificuldade de compreensão da sintaxe do paradigma orientado a objetos: "Mídia, eu acho um pouco mais complexo, a sintaxe é diferente e muda a linguagem, no Visualg é mais fácil, mas eu prefiro mídia porque, tipo, é mais complexo e dá para ter aquela noção que você está realmente fazendo aquilo. Você sente que está progredindo" (E3).

Um estudante mencionou dificuldade em relação ao idioma da linguagem e em relação aos conceitos matemáticos: "Eu tive mais dificuldade com questões que envolviam cálculos de matemática. Eu também tive dificuldade no inglês da linguagem” (E1).

Percebemos que a utilização de mídias é atraente, age como um facilitador na relação ensino-aprendizagem e atua diretamente na motivação. "Dá mais vontade de aprender, já que, como no primeiro ciclo foi feito e usa isso muito na internet, então eu acho mais interessante e fácil de aprender" (E4).

Ha um feedback rápido das atividades propostas pois a visualização é interativa: "Você vê a diferença, quando muda no código e vê a imagem alterando" (E4) e "Como eu acabei de falar, no caso, como está trabalhando com imagem que faz a modificação, tipo, mudar a cor, fica muito melhor do que ver somente texto no prompt de comando" (E2). Há também a possibilidade de aprender a programar em uma nova perspectiva lúdica, diferente do modelo tradicional, proporcionando aos estudantes momentos espontâneos e prazerosos na aquisição do conhecimento: "O uso de mídia é melhor, porque você pode aprender brincando, sabe, e no VisuAlg, é mais solução de problema” (E5).

\subsection{Aprendizagem de Conceitos de POO}

A metodologia utilizada seguiu o critério de aprender fazendo, com aulas teóricas onde eram apresentados conceitos de POO e atividades práticas intercaladas no mesmo dia. Ao final das aulas teóricas, eram propostos desafios e os estudantes procuravam resolver os 
VI Congresso Brasileiro de Informática na Educação (CBIE 2017)

Anais dos Workshops do VI Congresso Brasileiro de Informática na Educação (WCBIE 2017)

problemas navegando no ambiente do JES. Considerando a complexidade e abstração dos conceitos, as aulas foram planejadas de maneira que os exemplos sempre eram abordados fazendo analogias com a realidade.

Os resultados sugerem que programar utilizando manipulação de imagens torna mais intuitivo o aprendizado de conceitos relacionados ao paradigma orientado a objetos. Por exemplo, ao identificar uma classe: "Com o teórico dá para entender, só que para frisar mesmo realmente é melhor praticar e com a mídia facilitou” (E3), os atributos de uma classe “(...) e, com mídia, você vê a imagem e já sabe quais os atributos que ela tem" (E2) e como ela se comporta (i.e., métodos): "Quando chama o objeto para fazer o efeito, então você já pode fazer um método para cada efeito, a você sabe o que o método quer fazer" (E5). Surgiram alguns relatos de dificuldade na aprendizagem de alguns conceitos de $\mathrm{POO}$, no que tange à aquisição do conteúdo: "A maioria, eu tive dificuldade, porque, tipo, em uma aula eu pego o assunto e na outra eu já esqueço” (E4).

Linguagens orientadas a objetos apresentam um conceito característico importante: a herança, um mecanismo que permite a uma classe reaproveitar atributos e métodos em outra classe, gerando algumas vantagens. Por exemplo, a redução da necessidade de escrever códigos similares permite o reaproveitamento do código, reduzindo a quantidade de erros e duplicidade de atributos e métodos, além de diminuir o trabalho do programador. Os estudantes conseguiram identificar esta característica de forma natural: “(...) e ficar herdando o código, então você não precisa fazer o código novamente, acho que e a principal função da orientação a objetos, então herda um código já criado e pode usar para tudo" (E2).

\subsection{Engajamento}

O engajamento é um construto que se refere à relação entre indivíduo e atividade, atrelada ao contexto em que ela ocorre [Frydenberg et al. 2005]. O engajamento escolar trata de questões relacionadas sobre como os estudantes lidam com as tarefas propostas no âmbito escolar.

Ha três definições distintas na literatura para o engajamento: i) engajamento comportamental, que envolve a participação e o comprometimento dos estudantes nas atividades propostas no ambiente escolar e atividades extracurriculares, de tal modo que condutas afirmativas sejam executadas pelos estudantes; ii) engajamento emocional, relacionado com questões afetivas e emocionais dos estudantes diante das atividades e das pessoas que fazem parte do ambiente escolar; e iii) engajamento cognitivo, relacionado com a energia depositada pelos estudantes na atividade, não se contentando com o mínimo, indo além, sempre objetivando alcançar níveis mais elevados [Fredricks et al. 2004].

Durante as aulas, observamos como os estudantes se envolviam com os desafios, como se organizavam entre si para solucionarem os problemas, as discussões entre si, bem como as discussões dos estudantes com os tutores e monitores. Nossos resultados sugerem que houve um engajamento comportamental demonstrado na execução e concretização dos programas. Como exemplo, ao final das aulas, os estudantes sempre concluíam os desafios, demonstrando compreensão do objetivo do desafio proposto: "Ao final, todas as três duplas conseguiram concluir a atividade” (O1).

A programação em pares surgiu naturalmente logo nas primeiras aulas a partir da interação dos estudantes, solucionando dúvidas, discutindo possíveis soluções para 
VI Congresso Brasileiro de Informática na Educação (CBIE 2017)

Anais dos Workshops do VI Congresso Brasileiro de Informática na Educação (WCBIE 2017)

resolução das atividades. A partir daí, passamos a adotar este método como estratégia didática em todas as aulas.

Além dofeedback dos erros, os estudantes colocavam suas dúvidas para os tutores, que eram sempre sanadas: "O tutor vai até o aluno e verifica que foi erro de lógica, então indica a possível falha. E7 então corrige o código e, ao executar, funciona” (O2).

O engajamento cognitivo envolve o investimento do estudante na aprendizagem. Nas nossas observações, verificamos um engajamento cognitivo por parte de alguns estudantes, pois demonstraram um esforço empreendido em executar atividades que não eram propostas: "Além do programa da calculadora, E3 e E2 fizeram um programa criando a classe Boletim, com funções para calcular a média, demonstrando estarem motivados" (O1).

O estudo demonstrou que a manipulação de mídias, a programação em pares e a utilização do ambiente JES trouxeram respostas positivas nas ações e comportamentos dos estudantes, inclusive a partir de relatos com pretensões de continuar na área de computação: "Eu pretendo seguir na área de computação, porque eu gosto de programação e dá para criar coisas novas, dá para criar softwares novos e é algo que eu gosto. No caso, com a mídia dá para ver mais o que está fazendo, então me motivou mais ainda, no caso, o JES mesmo, até eu e E3 fizemos algumas coisas em casa...” (E2).

\section{Discussão}

Ao analisarmos a abordagem de ensino, percebemos que o uso de mídias, a linguagem Python e a ferramenta JES introduzem aspectos positivos e negativos. Os aspectos negativos relatados perpassam pela dificuldade encontrada no conhecimento da língua estrangeira (inglês) da ferramenta, além da dificuldade de assimilar as abstrações envolvidas. Porém, a maioria dos relatos das dificuldades encontradas não estão ligados à abordagem. As outras dificuldades estão associadas a diversos fatores como, por exemplo, a exigência de conhecimentos prévios matemáticos. Os aspectos positivos apontam para um maior envolvimento do estudante com o ambiente, principalmente pela possibilidade do feedback visual imediato. O sentimento dos estudantes é de que não era uma atividade cansativa, entediante, mas sim de "aprender brincando". O público é de adolescentes, então, a possibilidade de manipular imagens, criar efeitos em imagens e poder publicá-las nas redes sociais, permitiu um maior engajamento, tornando o caminho interessante em direção ao aprendizado.

Os conceitos de POO foram apresentados fazendo analogia com objetos reais, com a finalidade de facilitar a aprendizagem. Ao final das aulas teóricas, foram propostos desafios, e percebemos indícios de facilidade em relação à aprendizagem dos conceitos. Estes indícios foram respaldados através das nossas observações, dos relatos, ações e comportamentos. Observamos que os estudantes estavam totalmente envolvidos com as tarefas, pois abordaram tutores e monitores para esclarecer dúvidas, além de sempre trocarem ideias entre si. A maioria dos relatos revelou facilidade na compreensão, além da conclusão das atividades propostas. Ao solucionarem os desafios, os estudantes adquiriram competências e habilidades para desenvolver uma solução orientada a objetos.

$\mathrm{O}$ conceito de engajamento nos pareceu interessante para investigar a maneira como os estudantes desenvolvem suas atividades, pois corresponde à relação que eles 
estabelecem com as atividades que lhes são propostas em função do contexto. Esta relação é influenciada por fatores como: o estilo da atividade, a interação entre eles, a postura do professor e suas experiências anteriores.

Em várias oportunidades, percebemos a participação massiva dos estudantes quando desafiados, principalmente em: prestar atenção nas aulas, esclarecer dúvidas, concluir as atividades. Observamos uma interação crescente entre os estudantes, em que naturalmente passaram a programar em pares, fator que influenciou os resultados de maneira positiva, levando-nos, inclusive, a adotá-la como conduta padrão para a execução das atividades.

Atitudes positivas, como o esforço empreendido por alguns estudantes em buscar outras atividades extracurriculares para alcançar níveis mais elevados do conhecimento, não se contentando com o que foi trabalhado em sala de aula, demonstraram que eles não fizeram as atividades por obrigação, mas pelo anseio de aprender de fato os conceitos.

A partir desses fatores associados, percebemos que houve engajamento emocional, cognitivo e comportamental dos estudantes em relação às atividades, conceitos que estão intrinsecamente ligados: "Comportamento, emoção e cognição não são processos isolados, e sim fatores que se interrelacionam de maneira dinâmica" [Fredricks et al. 2004].

\section{Conclusões}

Este trabalho apresenta os resultados de um estudo de caso que analisa o impacto de uma abordagem de ensino de programação na aprendizagem dos estudantes do terceiro ano do Ensino Médio integrado com a Educação Profissional. Utilizamos a linguagem Python associada à ferramenta JES no contexto de manipulação de mídias.

Os resultados, baseados em uma pesquisa estritamente qualitativa, mostram que a abordagem utilizada, de fato, influenciou positivamente na aprendizagem dos conceitos do paradigma orientado a objetos, o que foi demonstrado através dos relatos, das discussões que surgiram no decorrer das aulas e da postura dos estudantes diante das atividades. Também nos permitiram perceber e interpretar os fatores que contribuíram para a aprendizagem. De fato, percebemos um maior engajamento comportamental, emocional e cognitivo dos estudantes em relação às atividades propostas.

Entender quais fatores contextuais interferem no engajamento dos estudantes e compreender como estes fatores colaboram para ou limitam o engajamento nas diversas circunstâncias de aprendizagem nos parece muito importante. Este entendimento permite decidir de maneira consciente e com maior segurança sobre quais estratégias serão utilizadas no contexto dos métodos de ensino e atividades a serem executadas, com objetivo de potencializar o engajamento dos estudantes.

Em trabalhos futuros, pretendemos realizar um estudo visando analisar a melhora nos níveis de engajamento dos estudantes nas atividades, considerando os inúmeros resultados de pesquisa que demonstram a ligação entre engajamento e aprendizagem.

\section{Referências}

Araujo, L. G. J., Santos, D. M. B., and Bittencourt, R. A. (2017). Ensino de Programação na Educação Básica através de Manipulação de Mídias. In COBENGE 2017 - XLV 
VI Congresso Brasileiro de Informática na Educação (CBIE 2017)

Anais dos Workshops do VI Congresso Brasileiro de Informática na Educação (WCBIE 2017)

Congresso Brasileiro de Educação em Engenharia, Joinville. Anais do XLV Congresso Brasileiro de Educação em Engenharia.

Bittencourt, R. A., Rocha, A. S., Santana, B. L., Santana, C. S., Carneiro, D. A., Borges, G. A., and Andrade, P. H. (2013). Aprendizagem de Programação Através de Ambientes Lúdicos em um curso de Engenharia de Computação: Uma Primeira Incursão. In XXI WEI - XXI Workshop sobre Educação em Computação, pages 749-758. Anais do XXXIII Congresso da Sociedade Brasileira de Computação.

Fredricks, J. A., Blumenfeld, P. C., and Paris, A. H. (2004). School Engagement: Potential of the Concept, State of the Evidence. Review of Educational Research, 74(1):59-109.

Frydenberg, E., Ainley, M., and Russell, V. (2005). Student Motivation and Engagement. Australia Dept of Education, Science and Training (DEST).

Grandell, L., Peltomäki, M., Back, R.-J., and Salakoski, T. (2006). Why Complicate Things?: Introducing Programming in High School Using Python. In ACE '06 Proceedings of the 8th Australasian Conference on Computing Education, volume 52, pages 71-80, Darlinghurst, Australia, Australia. Australian Computer Society, Inc.

Guzdial, M., Tew, A., Elliott, and Fowler, C. (2005). Tracking an Innovation in Introductory CS Education from a Research University to a Two-Year College. In SIGCSE '05 Proceedings of the 36th SIGCSE Technical Symposium on Computer Science Education, volume 37, pages 416-420.

Hinterholz, O. (2009). Tepequém: Uma Nova Ferramenta para o Ensino de Algoritmos nos Cursos Superiores em Computação. In WEI 2009 - XVII Workshop sobre Educação em Computação, volume 20, pages 485-488, Bento Gonçalves. Anais do XXIX Congresso da Sociedade Brasileira de Computação.

Oliveira, M. V., Rodrigues, L. C., and Queiroga, A. (2016). Material Didático Lúdico: Uso da Ferramenta Scratch para Auxílio no Aprendizado de Lógica da Programação. In WIE 2016 - XXII Workshop de Informática na Escola, volume 22, pages 359-368. Anais do XXII Workshop de Informática na Escola.

Papert, S. (1980). Mindstorms: Children, Computers, and Powerful Ideas. Basic Books, Inc., New York, NY, USA.

Quadros, J. R. T., Ogasawara, E., Amorim, M. C. M. S., and Ribeiro, R. C. (2012). Estudos sobre o Uso de Jogos para Apoiar o Aprendizado de Programação em um Curso Técnico de Informática. In IX SEGeT - Simpósio de Excelência em Gestão e Tecnologia, volume 20.

Santana, B. L., Figueredo, J. S. L., and Bittencourt, R. A. (2017). Motivação de Estudantes Non-Majors em uma Disciplina de Programação. In XXV WEI - XXV Workshop sobre Educação em Computação, São Paulo. Anais do XXXVII Congresso da Sociedade Brasileira de Computação.

Simon, B., Kinnunen, P., Porter, L., and Zazkis, D. (2010). Experience Report: CS1 for Majors with Media Computation. In ITiCSE'10 Proceedings of the Fifteenth Annual Conference on Innovation and Technology in Computer Science Education, pages 214 218. ACM New York, NY, USA. 